Brief Report

\title{
Alcohol-containing Mouthwashes and Oral Cancer: A Mechanistic Explanation
}

José Manuel Calderón-Montaño, Julio José Jiménez-Alonso, Emilio Guillén-Mancina, Estefanía Burgos-Morón and Miguel López-Lázaro*

Department of Pharmacology, Faculty of Pharmacy, University of Seville, Sevilla, Spain

*Correspondence to: Miguel López-Lázaro, Ph.D, Department of Pharmacology, Faculty of Pharmacy, University of Seville, C/ Profesor Garcia Gonzalez 2, 41012 Sevilla, Spain Tel.: +34 95455 63 48, E-mail: mlopezlazaro@us.es, https://orcid.org/0000-0003-2794-1647

\section{ABSTRACT}

Objective: To provide mechanistic evidence for the epidemiological link between long-term use of alcohol containing mouthwashes and oral cancer.

Methods: Human epithelial keratinocytes were exposed for 30 seconds to concentrations of ethanol commonly present in mouthwashes. After a recovery period, cell viability was assessed with the MTT assay.

Results: A marked cytotoxic effect was observed for ethanol concentrations of $20 \%$ and above.

Conclusions: The cytotoxicity of ethanol can explain the epidemiological association between mouthwash use and oral cancer. Recent findings indicate that the risk of developing cancer in a tissue is strongly determined by the number of stem cell divisions accumulated by the tissue during a person's lifetime; cell division is a major source of mutations and other cancer-promoting errors. Since cell death activates the division of stem cells, the cytotoxicity of ethanol on the cells lining the oral mucosa will promote the division of the stem cells located in deeper layers to produce new cells to regenerate the damaged epithelium. If we regularly use mouthwashes containing cytotoxic concentrations of ethanol, we will force the stem cells of the oral cavity to divide more often than usual and our risk of developing oral cancer will probably increase.

Clinical significance: Many mouthwashes contain percentages of ethanol above $20 \%$. Because ethanol is not crucial to prevent and reduce gingivitis and plaque, members of the dental team should consider the potential risk of oral cancer associated with frequent use of alcohol containing mouthwashes when advising their patients.

Keywords: cancer prevention; carcinogenesis; oral cancer; pharynx cancer; mouthrinses; ethanol.

\section{Introduction}

The association between alcohol-containing mouthwashes and the development of oral cancer has been a controversial topic over the last several decades. In 1979, a study based on 200 patients with oral cancers showed that 10 of the 11 patients who were non-smokers and non-drinkers had 
used mouthwash many times daily for more than 20 years; most of them used a brand of mouthwash containing 25\% alcohol [1]. Prompted by this clinical observation, several case-control studies were carried out. Two studies published in leading cancer journals showed results supporting a positive association between alcohol-containing mouthwashes and oral cancer [2,3]. One of these investigations revealed that the risk generally increased in proportion to the duration and frequency of mouthwash use, and that the increased risk was confined to users of mouthwashes high in alcohol [3]. Subsequent epidemiological studies reported mixed results. A meta-analysis of these studies published in 2012 [4] showed a positive but non-statistically significant association between regular mouthwash use and risk of oral cancer ( $R R=1.13 ; 95 \%$ CI $0.95,1.35)$. A similar association was found for mouthwashes containing $25 \%$ of alcohol $(R R=1.16$; $95 \%$ CI $0.44,3.08)$. No significant trend in risk was found with increased daily mouthwash use [4]. To provide more definitive evidence on the presence or absence of an association between mouthwash use and oral cancer, the International Head and Neck Cancer Epidemiology (INHANCE) Consortium conducted a large pooled analysis to overcome the limitations of metaanalyses [5]. The INHANCE Consortium has experience in the evaluation of risk factors for head and neck cancers. The results, published in 2016, showed that the odds ratios (OR) for cancers of the oral cavity and oropharynx were 1.11 (95\% CI 1.00, 1.23) and 1.28 (95\% CI 1.06, 1.56). The risk also increased with the duration and frequency of use of mouthwash; the OR was 1.26 (95\% CI 0.98, 1.62) for people who used mouthwash more than once per day, and 1.28 (95\% CI 1.06, 1.56) for those who used it during 36 years or longer [5]. In simple terms, these data mean that if the number of people diagnosed with oral cancer in a population not using mouthwashes is 100, the number would be 126 or 128 if the same population uses mouthwashes regularly. The risk of oral cavity cancers associated with mouthwash use is similar to the risk of these cancers associated with the consumption of one alcoholic drink per day [6]. Although a causal link between mouthwash use and oral cancer cannot be established from the epidemiological data, the INHANCE Consortium concluded that there are potential risks for oral cavity cancers in long-term and frequent users of mouthwash [5].

The mechanism by which alcohol-containing mouthwashes can increase the risk of oral cancer is not defined. Although alcohol consumption is a known risk factor for cancer [6,7], it is widely accepted that ethanol must be transformed into the mutagenic metabolite acetaldehyde in order to cause cancer (ethanol is not mutagenic). However, most acetaldehyde derived from alcohol consumption is produced in the liver and then distributed throughout the body, and the alcohol of mouthwashes does not reach the liver unless ingested. Oral bacteria can metabolize ethanol into acetaldehyde; however, the amount of acetaldehyde produced by oral bacteria is relatively low, and the antibacterial activity of mouthwashes partially prevents this metabolic transformation [8]. Although the administration of alcohol has been reported to cause acetaldehyde-DNA adducts in the oral mucosa, the disappearance of these DNA adducts several hours after the administration of alcohol suggests that these DNA alterations are repaired [9]. In addition, the possible formation of permanent acetaldehyde-mediated DNA alterations in the cells lining the oral mucosa would be eliminated when these cells are replaced by new cells during tissue renewal. We cannot forget that carcinogenesis requires the multistep accumulation of DNA changes over years or decades, and that the cells lining the oral cavity are typically replaced every few weeks [10,11]. Lack of biological plausibility is a barrier to accept the epidemiological link between long-term and frequent use of 
mouthwashes and oral cavity cancers. Without a mechanistic explanation, the epidemiological evidence of carcinogenicity may be attributed to bias, confounding or chance, and taking preventive measures may be considered unnecessary.

We recently proposed that the cytotoxicity of ethanol on the cells lining the oral cavity, pharynx and esophagus can explain why alcohol consumption preferentially exerts a local carcinogenic effect $[12,13]$. Using a similar approach, here we provide evidence that the potential risk of oral cavity cancers associated with regular mouthwash use can be explained by the presence of cytotoxic concentrations of ethanol in most mouthwashes.

\section{Materials and Methods}

The human keratinocyte cell line HaCaT (Cell Line Service; L\#300493-4212) was grown in Dulbecco's Modified Eagle's Medium (DMEM) at $37^{\circ} \mathrm{C}$ in a humidified atmosphere containing $5 \%$ CO2. DMEM was supplemented with $100 \mathrm{U} / \mathrm{mL}$ penicillin, $100 \mu \mathrm{g} / \mathrm{mL}$ streptomycin and $10 \%$ fetal bovine serum. Cell culture reagents were obtained from Thermo Fisher Scientific. Ethanol absolute $(\geq 99.8 \%$, AnalaR NORMAPUR, ACS, Reag. Ph. Eur.) was obtained from VWR Chemicals.

Exponentially growing cells were seeded into 96-well plates. Once the cells formed a monolayer, $100 \mu \mathrm{L}$ of PBS containing several concentrations of ethanol (5\%, 10\%, 15\%, 20\%, 25\% and $30 \%$ ) were added to the wells; 2 wells were used for each ethanol concentration. The ethanol solutions were removed 30 seconds later. After a a 20-h recovery period in DMEM medium, cell viability was estimated with the MTT assay. This colorimetric technique is based on the ability of viable cells to transform the MTT salt (3-(4,5-dimethylthiazol-2-yl)-2,5-diphenyltetrazolium bromide) into a purple formazan product. After removing the DMEM medium, $125 \mu \mathrm{L}$ MTT ( $1 \mathrm{mg} / \mathrm{mL}$ in medium) were added to each well. The plates were incubated for 2 hours to allow viable cells to transform the MTT compound into the formazan product. This insoluble product was solubilized by adding $80 \mu \mathrm{L} 20 \%$ SDS in $0.02 \mathrm{M} \mathrm{HCl}$ to each well and by incubating the plates overnight at $37^{\circ} \mathrm{C}$. Finally, optical densities were measured at $540 \mathrm{~nm}$ on a multi-well plate spectrophotometer reader. Cell viability was expressed as percentage in relation to controls $[14,15]$. Data were averaged from at least three independent experiments. Data are shown as means \pm standard error of the mean (SEM). Paired, two-tailed, $t$-test was used for statistical analysis. A $P$-value $<0.05$ is considered statistically significant and is indicated with an asterisk $\left(^{*}\right)$, a $P$-value $<0.01$ is indicated with a double asterisk $\left.{ }^{* *}\right)$, and a $P$-value $<0.001$ is indicated with a triple asterisk $\left.{ }^{(* *}\right)$.

\section{Results and Discussion}

Since keratinocytes are the most common cell type lining the oral mucosa [10], we used a human keratinocyte cell line for our experiments. To simulate the 30-second rinse recommended in most mouthwash instructions, the cells were exposed to different concentrations of ethanol for 30 seconds. After a recovery period of 20 hours, we estimated cell viability with the MTT assay. Results show a clear cytotoxic effect at concentrations of ethanol of $20 \%$ and above (Figure 1). Marked cytotoxic effects were also observed after a 10-second exposure to ethanol 20\% (results not shown). The cytotoxic effect of ethanol $15 \%$ was very mild after a 30 -second exposure, but increased 
notably at longer exposure times (i.e., 60 seconds and 5 minutes; results not shown). No cytotoxicity was observed when the cells were exposed to ethanol 10\% during 5 minutes (results not shown). Figure 1 shows that a 30-second exposure to concentrations of ethanol commonly present in mouthwashes induces a marked cytotoxic effect on human epithelial keratinocytes.

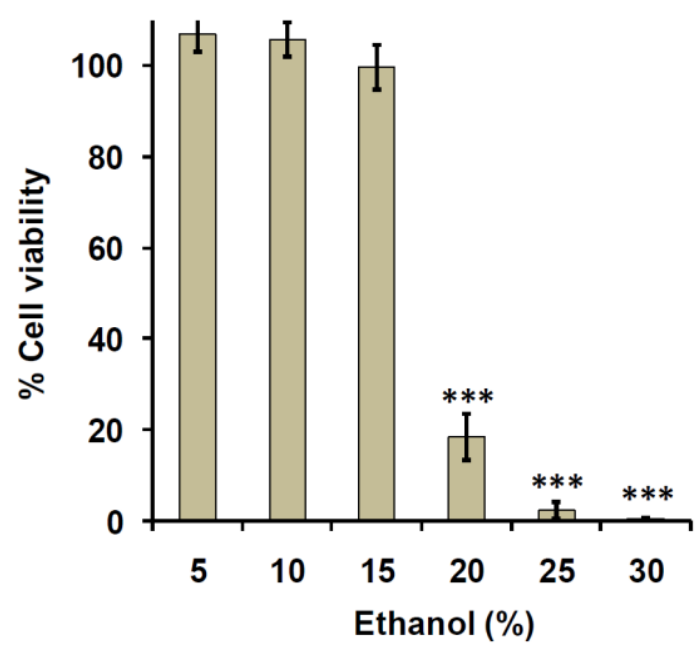

Figure 1. Human epithelial keratinocytes (HaCaT cells) were exposed for 30 seconds to a solution of PBS containing different ethanol concentrations. After a recovery period of 20 hours in ethanol-free medium, cell viability was estimated with the MTT assay.

The cytotoxicity of ethanol can explain the epidemiological association between mouthwash use and cancer of oral cavity and oropharynx [5]; note that the oropharynx is highly exposed to mouthwashes during gargling (a common practice in mouthwash users). Recent findings indicate that the risk of developing cancer in a tissue is strongly determined by the number of stem cell divisions accumulated by the tissue during a person's lifetime [16,17]. As discussed elsewhere, cell division is a major source of mutations $[16,17]$ and other cancer-promoting errors $[11,18]$. Once our body tissues are formed and we stop growing, cell death occurring during tissue renewal is the main cause for the division of stem cells. This explains why most cancers occur in self-renewing tissues and why cancer incidence increases dramatically with age in these tissues [11]. Persistent exposure to pathological or environmental cytotoxic factors will also promote tissue renewal and cancer formation. These factors do not need to cause an acute cytotoxic effect to increase the risk of cancer; they just need to cause sufficient damage to reduce the lifespan of the cells that need to be replaced by stem cells [11]. Since cell death is a major trigger for the division of stem cells, the cytotoxicity of ethanol on the cells lining the oral cavity will promote the division of the stem cells located in deeper layers to produce new cells to regenerate the damaged epithelium. If we regularly use mouthwashes containing cytotoxic concentrations of ethanol, we will force the stem cells of the oral cavity to divide more often than usual, and they will accumulate an extra number of DNA alterations that will increase our risk of developing oral cancer.

Most mouthwashes contain alcohol, typically in the order of 20\% [5]. Essential oil mouthwashes generally contain the highest ethanol concentrations (over 20\%). Ethanol has several roles in 
mouthrinses; in addition to having a preservative effect, it helps dissolve essential oils and deliver them into the plaque-biofilm matrix. However, the presence of ethanol in mouthwashes is not necessary to prevent and reduce gingivitis and plaque. Leading mouthwash brands also offer ethanol-free variants that contain other solubilizing and preservative agents that perform a similar role to alcohol. Members of the dental team should be aware of the potential risk of oral cancer associated with frequent use of alcohol containing mouthwashes when advising their patients. Mouthwashes should be labeled with the concentration of alcohol so that users can make informed choices; currently, finding the ethanol concentration of some mouthwashes is difficult.

\section{Conflicts of interest}

The authors declare no conflict of interest.

\section{References}

1. Weaver A, Fleming SM, Smith DB. Mouthwash and oral cancer: carcinogen or coincidence? J Oral Surg 1979; 37(4):250-253.

2. Wynder EL, Kabat G, Rosenberg S, Levenstein M. Oral cancer and mouthwash use. J Natl Cancer Inst 1983; 70(2):255-260.

3. Winn DM, Blot WJ, McLaughlin JK, Austin DF, Greenberg RS, Preston-Martin S, Schoenberg JB, Fraumeni JF, Jr. Mouthwash use and oral conditions in the risk of oral and pharyngeal cancer. Cancer Res 1991; 51(11):3044-3047.

4. Gandini S, Negri E, Boffetta P, La Vecchia C, Boyle P. Mouthwash and oral cancer risk quantitative meta-analysis of epidemiologic studies. Ann Agric Environ Med 2012; 19(2):173-180.

5. Boffetta P, Hayes RB, Sartori S, Lee YC, Muscat J, Olshan A, Winn DM, Castellsague X, Zhang ZF, Morgenstern H, Chen C, Schwartz SM, Vaughan TL, Wunsch-Filho V, Purdue M, Koifman S, Curado MP, Vilensky M, Gillison M, Fernandez L, Menezes A, Daudt AW, Schantz S, Yu G, D'Souza G, Haddad RI, La Vecchia C, Hashibe M. Mouthwash use and cancer of the head and neck: a pooled analysis from the International Head and Neck Cancer Epidemiology Consortium (INHANCE). Eur J Cancer Prev 2016; 25(4):344-348.

6. Bagnardi V, Rota M, Botteri E, Tramacere I, Islami F, Fedirko V, Scotti L, Jenab M, Turati F, Pasquali E, Pelucchi C, Galeone C, Bellocco R, Negri E, Corrao G, Boffetta P, La Vecchia C. Alcohol consumption and site-specific cancer risk: a comprehensive dose-response metaanalysis. Br J Cancer 2015; 112(3):580-593.

7. Connor J. Alcohol consumption as a cause of cancer. Addiction 2017; 112(2):222-228.

8. Moazzez R, Thompson H, Palmer RM, Wilson RF, Proctor GB, Wade WG. Effect of rinsing with ethanol-containing mouthrinses on the production of salivary acetaldehyde. Eur J Oral Sci 2011; 119(6):441-446. 
9. Balbo S, Meng L, Bliss RL, Jensen JA, Hatsukami DK, Hecht SS. Kinetics of DNA adduct formation in the oral cavity after drinking alcohol. Cancer Epidemiol Biomarkers Prev 2012; 21(4):601-608.

10. Squier CA, Kremer MJ. Biology of oral mucosa and esophagus. J Natl Cancer Inst Monogr 2001;(29):7-15.

11. Lopez-Lazaro M. The stem cell division theory of cancer. Crit Rev Oncol Hematol 2018; 123:95-113 DOI: https://doi.org/10.1016/j.critrevonc.2018.01.010.

12. Lopez-Lazaro M. A local mechanism by which alcohol consumption causes cancer. Oral Oncol 2016; 62:149-152.

13. Guillen-Mancina E, Calderon-Montaño JM, Lopez-Lazaro M. Avoiding the ingestion of cytotoxic concentrations of ethanol may reduce the risk of cancer associated with alcohol consumption. Drug Alcohol Depend 2017; 183:201-204

14. Calderon-Montano JM, Burgos-Moron E, Orta ML, Pastor N, Austin CA, Mateos S, LopezLazaro M. Alpha, beta-unsaturated lactones 2-furanone and 2-pyrone induce cellular DNA damage, formation of topoisomerase I- and II-DNA complexes and cancer cell death. Toxicol Lett 2013; 222(1):64-71.

15. Riss TL, Moravec RA, Niles AL, Benink HA, Worzella TJ, Minor L, Riss TL, Moravec RA, Niles AL. Cell Viability Assays Cytotoxicity testing: measuring viable cells, dead cells, and detecting mechanism of cell death. Methods Mol Biol 2011; 740:103-14.

16. Tomasetti C, Vogelstein B. Cancer etiology. Variation in cancer risk among tissues can be explained by the number of stem cell divisions. Science 2015; 347(6217):78-81.

17. Tomasetti C, Li L, Vogelstein B. Stem cell divisions, somatic mutations, cancer etiology, and cancer prevention. Science 2017; 355(6331):1330-1334.

18. Lopez-Lazaro M. Cancer etiology: Variation in cancer risk among tissues is poorly explained by the number of gene mutations. Genes Chromosomes Cancer 2018; DOI: 10.1002/gcc.22530:10. 